\title{
Biomarkers for Early Detection of Hypertensive Disorders in Pregnancy: Current Applications and Future Directions- The Role of Extracellular Matrix
}

\author{
N Popovski ${ }^{1}$ and A Nikolov*2 \\ ${ }^{1}$ Department of Obstetrics and Gynaecology, Bulgaria \\ ${ }^{2}$ Institute for Scientific Research, Bulgaria \\ *Corresponding author: Asparuh Nikolov, Institute for scientific research, 5800 Pleven, Bulgaria
}

\section{ARTICLE INFO}

Received: March 14, 2019

Published: March 20, 2019

Citation: N Popovski, A Nikolov. Biomarkers for Early Detection of Hypertensive Disorders in Pregnancy: Current Applications and Future Directions- The Role of Extracellular Matrix. Biomed J Sci \& Tech Res 16(2)-2019. BJSTR. MS.ID.002816.

Keywords: Hypertensive Disorders In Pregnancy; Biomarkers; Extracellular Matrix

\section{ABSTRACT}

Hypertensive disorders in pregnancy (HDP) are the most common medical complications, affecting $5-10 \%$ of pregnancies worldwide. Their early detection is critical for risk stratification and prevention of further pregnancy complications. Despite current intensive search of biomarkers for early detection and prognosis of HDP, the clinical efficacy of these markers has shown low predictive value. There is a growing evidence for extracellular matrix involvement in HDP's pathophysiology. Clinicians need better tools for predicting HDP. Biomarkers of extracellular matrix turnover are one of the possible candidates.

Abbreviations: HDP: Hypertensive Disorders In Pregnancy; PE: Preeclampsia; MMP: Matrix Metallo Proteinases; ECM: Extra Cellar Matrix; TIMPs: Tissue Inhibitor of Matrix Metalloproteinases; VEGF: Vascular Endothelial Growth Factor; RUPP: Reduced Uteroplacental Perfusion Pressure; VEGF: Vascular Endothelial Growth Factor; RUPP: Reduced Uterine Perfusion Pressure; HTN-Preg: Hypertensive-Pregnancy

\section{Introduction}

Hypertension is the most common medical problem encountered during pregnancy, complicating 2-3\% of pregnancies. Hypertension in pregnancy is not a single entity but comprises preexisting hypertension, gestational hypertension, pre-eclampsia, pre-existing hypertension plus superimposed gestational hypertension with proteinuria and antenatally unclassifiable hypertension [1]. Preeclampsia (PE) is a disease, which has not been fully studied yet. It is a major cause of maternal and perinatal morbidity and mortality [2]. Preeclampsia is a prevalent and ambigous disease, in part characterized by poor remodelling of the spiral arteries. However, preeclampsia does not always clinically present when remodelling has failed to occur [3]. Biomarkers of early preeclampsia detection are critical for risk stratification and testing therapies [4].Traditionally preeclampsia biomarkers are grouped into the next categories [5]: a) Renal dysfunction- microalbuminuria, serum uric acid, urinary calcium excretion.

b) Fetoplacental unit endocrinology and vascular resistance and perfusion dysfunction- human chorionic gonadotropin, pregnancy associated plasma protein $\mathrm{A}$, adiponectin, mean blood pressure, Doppler ultrasound, rennin, vasopressin.

c) Endothelial dysfunction, growth factors and oxidative stress- prostacyclin, tromboxane, cytokines, vascular endothelial growth factor, isoprostanes apolipoprotein E, placenta growth factor, soluble FMS tyrosine kinase, soluble endoglin.

These molecules have shown low predictive values as individual biomarkers. Moreover, the sensitivity of a single biomarker tends to be very low. When a combination model is used, the predictive 
value is higher. Therefore, there is an urgent need for high quality, novel research in biomarkers for PE, so that the best predictive strategy can be identified in order to improve the management of women in high risk for development PE.

\section{Findings}

Traditional concept of PE biomarkers did not include one main pathophysiology link- extracellar matrix (ECM) turnover. Usually ECM is thought to be a relatively inactive structure, which is playing a role of cells and vessels' scaffold. Recent data provides evidence that ECM is a dynamic and metabolically active structure [6]. ECM turnover is regularized mainly by matrix metalloproteinases (MMP) and tissue inhibitor of matrix metalloproteinases (TIMPs) [7]. Changes in ECM are suspected of contributing to the genesis and progression of hypertensive disorders of pregnancy. "Matrix metalloproteinases (MMPs) are important regulators of vascular and uterine remodeling. Increases in MMP-2 and MMP-9 have been implicated in vasodilation, placentation, and uterine expansion during normal pregnancy. The increases in MMPs could be induced by the increased production of estrogen and progesterone during pregnancy. MMP expression/activity may be altered during complications of pregnancy. Decreased vascular MMP-2 and MMP9 may lead to decreased vasodilation, increased vasoconstriction, hypertensive pregnancy, and preeclampsia.

These circulating factors could target MMPs in the extracellular matrix as well as endothelial and vascular smooth muscle cells, causing generalized vascular dysfunction, increased vasoconstriction and hypertension in pregnancy. MMP activity can also be altered by endogenous TIMPs and changes in the MMP/TIMP ratio. In addition to their vascular effects, decreases in expression/activity of MMP-2 and MMP-9 in the uterus could impede uterine growth and expansion and lead to premature labor Understanding the role of MMPs in uteroplacental and vascular remodeling and function could help design new approaches for prediction and management of preeclampsia and premature labor" [8]. Authors Narumiya $\mathrm{H}$ et al. hypothesized that MMP levels are increased in women with preeclampsia. They used a model with human umbilical vein endothelial cells in response to vascular endothelial growth factor (VEGF); (0.1-10 ng/mL). Data showed that plasma MMP-2 levels were significantly higher in women with preeclampsia $(n=12)$ compared to women with uncomplicated pregnancies ( $\mathrm{n}=12)$; (arbitrary intensity units: $690 \pm 111$ and 252 \pm 56 , respectively, $\mathrm{p}<0.05$ ).

MMP-9 levels were below the level of detection. In addition, VEGF stimulated endothelial MMP-2 and MMP-9 release in a concentration- and time-dependent (6-24 h) manner. Moreover, VEGF stimulation of MMP release occurs without significantly affecting the release of TIMP-1 and TIMP-2. These data suggest that VEGF promotes secretion of MMPs from endothelial cells that, in turn, could alter vascular function in women with preeclampsia
[9]. Authors Myer JE, also examine ECM biomarkers as potential molecules for predicting preeclampsia. They determined levels of matrix metalloproteinase MMP-2 and MMP-9, and TIMP-1 and TIMP-2 in plasma of women destined to develop preeclampsia prior to the onset of clinical disease. "Plasma samples were taken from women whose pregnancies were subsequently complicated by preeclampsia and from normal pregnant women at 22 and 26 weeks and at delivery or diagnosis. Following equal protein loading, MMP- 2 and 9 and TIMP-1 and 2 were quantified using zymography and Western blot analysis, respectively. At all three gestational time points an imbalance in the MMP-2:TIMP-1 ratio was found in patients who subsequently developed preeclampsia.

Authors speculated that increased net MMP-2 activity may contribute to the endothelial dysfunction that is central to the pathophysiology of preeclampsia" [10]. In 2014 Li W, tested the role of MMPs in hypertensive-pregnancy (HTN-Preg), maternal and fetal parameters, MMPs expression, activity and distribution, and collagen and elastin content were measured in uterus, placenta and aorta of Norm-Preg rats and in rat model of reduced uteroplacental perfusion pressure (RUPP). "Western blots and gelatin zymography revealed decreases in amount and gelatinase activity of MMP-2 and MMP-9 in uterus, placenta and aorta of RUPP compared with Norm-Preg rats. Immunohistochemistry confirmed reduced MMPs in uterus, placenta and aortic media of RUPP rats. Collagen, but not elastin, was more abundant in uterus, placenta and aorta of RUPP than Norm-Preg rats. The anti-angiogenic factor soluble fms-like tyrosine kinase-1 (sFlt-1) decreased MMPs in uterus, placenta and aorta of Norm-Preg rats, and vascular endothelial growth factor (VEGF) reversed the decreases in MMPs in tissues of RUPP rats.

Thus, placental ischemia and anti-angiogenic sFlt-1 decrease uterine, placental and vascular MMP-2 and MMP-9, leading to increased uteroplacental and vascular collagen, and growthrestrictive remodeling in HTN-Preg". Investigators concluded that angiogenic factors and MMP activators may reverse the decrease in MMPs and enhance growth-permissive remodeling in preeclampsia" [11]. In 2017 the same authors measured MMP levels and distribution in the aorta, uterus, and placenta of normal pregnant (Preg) rats and pregnant rats with reduced uterine perfusion pressure (RUPP). "Maternal blood pressure was higher and the litter size and pup weight were lower in RUPP compared with Preg rats. Gelatin zymography showed prominent uterine MMP-2 and MMP-9 activity that was dependent on the amount of loaded protein. At saturating protein loading, both gelatin and casein zymography revealed two additional bands corresponding to MMP-1 and MMP-7 that were greater in the aorta, uterus, and placenta of RUPP compared with Preg rats. Western blots and immunohistochemistry confirmed increased MMP-1 and MMP7 in the aorta, uterus, and placenta of RUPP versus Preg rats. The levels of MMP-1 and MMP-7 substrate collagen type I were greater in tissues of RUPP compared with Preg rats" [12]. 
These findings implacably raise the question for pathological collagen type I turnover in hypertensive disorders of pregnancy. Collagens are large family of proteins with more than 20 different collagen types identified so far. They are centrally involved in the formation of fibrillar and microfibrillar networks of the extracellular matrix, basement membranes as well as other structures of the extracellular matrix [13]. The latter studies show an evidence for pathological ECM turnover and particularly altered collagen type I metabolism in hypertensive disorders of pregnancy.

\section{Conclusion}

Preeclampsia is a complication of pregnancy manifested as maternal hypertension and often fetal growth restriction. Despite the active search of multiple potential PE biomarkers, clinical efficacy of these markers has shown low predictive value. There has not been discovered a single biomarker predicting PE yet. However, a combination model could possibly perform better than a single molecule, eventually adding markers of ECM turnover. According to us the most appropriate strategy would probably be an integrative model using ECM biomarkers and uterine artery Doppler assessment. We expect our review to turn investigators" attention for the possible role of extracellular matrix turnover in the pathophysiology of PE. Larger studies are needed to clarify the possible use of ECM biomarkers as PE predictive markers, eventually in combination with echographic examination for better sensitivity and specificity.

\section{References}

1. (2018) ESC Guidelines for the management of cardiovascular diseases during pregnancy. European Heart Journal 39(34): 3165-3241.

2. Gallo M, Wright D, Casanova C, Campanero M, Nicolaides K (2016) Competing risks model in screening for preeclampsia by maternal factors and biomarkers at 19-24 weeks' gestation. American Journal of Obstetrics and Gynecology 214(5): 6191-6197.

3. Ferguson KK, Meeker JD, McElrathTF, Mukherjee B, Cantonwine DE (2017) Repeated measures of inflammation and oxidative stress biomarkers in preeclamptic and normotensive pregnancies. American Journal of Obstetrics and Gynecology 216(5): 5271-5279.

4. Karumanchi SA, Granger JP (2015) Preeclampsia and Pregnancy-Related Hypertensive Disorders. Hypertension 67: 238-242.

5. Carty DM, Delles C, Dominiczak AF (2008) Novel Biomarkers for Predicting Preeclampsia. Trends Cardiovasc Med18 (5-24): 186-194.

6. Gerd Heusch, Peter Libby, Bernard Gersh, Derek Yellon, Michael Böhm, et al. (2014) Lancet Seminar: Cardiovascular Remodelling in Coronary Artery Disease and Heart Failure. The Lancet. 383(9932): 1933-1943.

7. Klein T, Bischoff R (2011) Physiology and pathophysiology of matrix metalloproteases. Amino Acids 41(2): 271-290.

8. Chen J, Khalil RA (2017) Chapter Four-Matrix Metalloproteinases in Normal Pregnancy and Preeclampsia. Progress in Molecular Biology and Translational Science 148: 87-165.

9. Narumiya H, Zhang Y, Fernandez Patron C, Guilbert LJ, Davidge ST (2001) Matrix metalloproteinase-2 is elevated in the plasma of women with preeclampsia. Hypertens Pregnancy 20(2): 185-194.

10. Myers JE, Merchant SJ, Macleod M, Mires GJ, Baker PN, et al. (2005) MMP2 levels are elevated in the plasma of women who subsequently develop preeclampsia. Hypertens Pregnancy 24(2): 103-15.

11. Li W, Mata KM, Mazzuca MQ, Khalil RA (2014) Altered matrix metalloproteinase-2 and - 9 expression/activity links placental ischemia and anti-angiogenic sFlt-1 to uteroplacental and vascular remodeling and collagen deposition in hypertensive pregnancy. Biochem Pharmacol 89: 370-385.

12. Li W, Cui N, Mazzuca MQ Mata KM, Khalil RA (2017) Increased vascular and uteroplacental matrix metalloproteinase-1 and -7 levels and collagen I deposition in hypertension-in-pregnancy. Role of TNF-a. Am J Physiol Heart Circ Physiol.

13. Gelse K, Pöschl E, Aigner T (2003) Collagens-structure, function, and biosynthesis. Adv Drug Deliv Rev 55: 1531-1546.

\section{ISSN: 2574-1241}

DOI: 10.26717/BJSTR.2019.16.002816

Asparuh Nikolov. Biomed J Sci \& Tech Res

This work is licensed under Creative Commons Attribution 4.0 License

Submission Link: https://biomedres.us/submit-manuscript.php

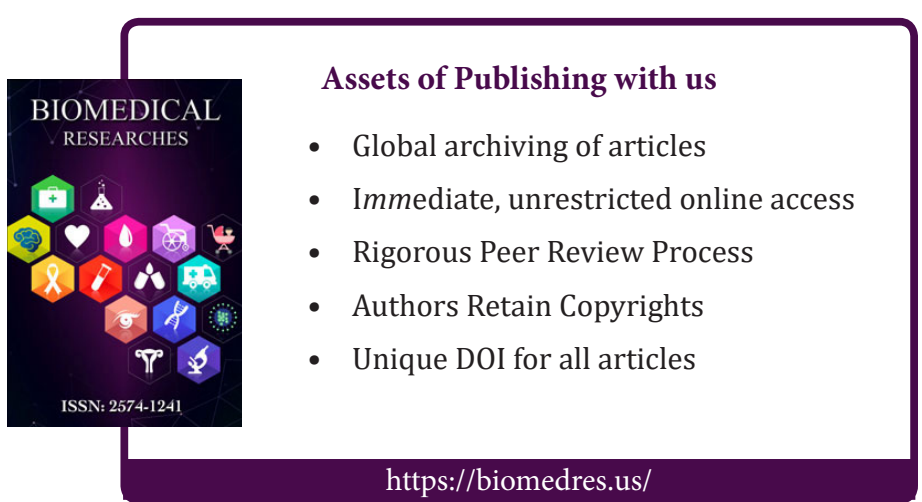

